\title{
Utilidad de isosorbide sublingual en la reversibilidad de la hipertensión pulmonar reactiva, en pacientes candidatos a trasplante cardíaco
}

\author{
Fernando Baraona R, Pablo Castro G, 0 svaldo Pérez P, \\ Sergio Morán V, Ricardo Zalaquett S, \\ Manuel J Irarrázabal L, Jorge Jalil M. \\ U sefulness of sublingual isosorbide \\ to assess the reversibility of \\ pulmonary hypertension \\ in cardiac transplant candidates
}

Background: Continuous infusion of short life vasodilators are employed to test reversibility of pulmonary hypertension in cardiac transplant candidates. Sublingual isosorbide administration has not been described in the literature and it might be a simpler alternative. Aim: To evaluate sublingual isosorbide administration as a test of reversibility of pulmonary hypertension in heart failure. Patients and Methods: Prospective evaluation of patients referred for cardiac transplant evaluation. Patients underwent right catheterization for hemodynamic measurements at baseline and after repeated doses of $5 \mathrm{mg}$ sublingual isosorbide every 5 minutes until observing a decrease in pulmonary vascular resistance decrease or symptomatic hypotension. Results: Twenty one patients, 18 men, age $49 \pm 15$ years, were studied. Fourteen (66\%) were transplanted. The mean sublingual isosorbide dose was $15 \pm 5 \mathrm{mg}$. After isosorbide administration, there was a significant decrease in mean arterial pressure ( $80 \pm 8.5$ to $71 \pm 6.6 \mathrm{mmHg}, \mathrm{p} \varangle 0.0001)$, mean pulmonary artery pressure ( $38 \pm 11$ to $26 \pm 7.8 \mathrm{mmHg}, \mathrm{p} \varangle 0.0001)$, systemic vascular resistance ( $1540 \pm 376$ to $1277 \pm 332$ dyn* $/ \mathrm{cm} 5 \mathrm{p} \varangle 0.001)$, pulmonary vascular resistance (3.5 \pm 2.2 to $2,5 \pm 1.6$ Wood Units, $\mathrm{p} \varangle 0.05)$ and transpulmonary gradient $(13 \pm 7$ a $10 \pm 4 \mathrm{mmHg}, \mathrm{p}<0.004)$. The cardiac output increased from $3.96 \pm 0.7$ to $4.38 \pm 0.9 \mathrm{~L} / \mathrm{min}, \mathrm{p}=0.05$. The relation between pulmonary and systemic vascular resistance before and after isosorbide was 0.17 and 0.15 , respectively $(\mathrm{p}=0.04)$. One transplanted patient with partial reversibility of pulmonary hypertension developed acute right heart failure. Conclusions: Sublingual isosorbide administration is useful and well tolerated to evaluate the reversibility of pulmonary hypertension prior cardiac transplant (Rev Méd Chile 2006; 134: 201-6).

(Key w ords: Heart transplantation; Isosorbide; Hypertension, pulmonary)

Recibido el 5 de enero, 2005. Aceptado el 16 de junio, 2005.

Departamento de Enfermedades Cardiovasculares, Hospital Clínico Pontifica Universidad Católica de Chile, Santiago Chile.

Correspondencia a: Dr. Pablo Castro. Departamento de Enfermedades Cardiovasculares, Pontificia Universidad Católica de Chile. Marcoleta 347, Santiago, Chile. E mail: pcastro@med.puc.cl 
$\mathrm{E}^{1}$ trasplante cardíaco es el tratamiento de elección para aquellos pacientes con insuficiencia cardíaca refractaria a terapia médica, en cuanto a mejoría de sobrevida y calidad de vida ${ }^{1}$. Actualmente, la indicación más frecuente corresponde a pacientes portadores de cardiopatía coronaria.

En la evaluación de los posibles candidatos es necesaria la realización de sondeo cardíaco derecho, porque permite determinar la resistencia vascular pulmonar (RVP), que es el principal criterio hemodinámico. Si la RVP es mayor a 4 unidades Woods (UW), contraindica el trasplante cardíaco, ya que es un predictor de insuficiencia cardíaca derecha y de mortalidad precoz posterior al trasplante ${ }^{2}$. Asimismo, la insuficiencia cardíaca derecha corresponde a 50\% de las complicaciones cardíacas y a $20 \%$ de las muertes tempranas post trasplante ${ }^{3,4}$.

Se ha observado que la reversibilidad de la hipertensión pulmonar demostrada con medios farmacológicos (mediante la administración de vasodilatadores endovenosos de vida media corta) en algunos pacientes se correlaciona con una buena evolución post trasplante ${ }^{5}$. La hipertensión pulmonar severa e irreversible constituye una exclusión de candidatos a trasplante, a no ser que se considere el trasplante de corazón-pulmón 6 .

La mayoría de los pacientes con insuficiencia cardíaca avanzada secundaria a miocardiopatía isquémica 0 dilatada, tienen un componente reactivo en la resistencia vascular pulmonar elevada, debido a la presión elevada de la aurícula izquierda. Con el objetivo de determinar la reversibilidad de RVP, dentro del protocolo de evaluación de los candidatos a trasplante, se administran vasodilatadores intravenosos (nitroprusiato de sodio, nitroglicerina y prostaglandinas), agentes inhalatorios como el óxido nítrico, e inótropos intravenosos (milrinona, levosimendan, dobutamina) ${ }^{7-11}$. Por otro lado, la respuesta hemodinámica durante estas pruebas, evaluada por la presión arterial sistémica, también constituye un indicador de mortalidad post trasplante. Esto quedó demostrado en un estudio en el que, sólo a expensas de hipotensión sintomática, se logró disminuir la RVP y se asoció a menor sobrevida ${ }^{12}$.

El uso de isosorbide sublingual para disminuir la RVP no está descrito en la literatura y podría ser una alternativa más económica y simple de utilizar (por parte del equipo médico y de enfermería) en estos pacientes.

El objetivo del presente trabajo es evaluar la acción de isosorbide sublingual como prueba de reversibilidad de hipertensión pulmonar secundaria a falla cardíaca.

\section{METOdologíA}

Se evaluó en forma prospectiva a los pacientes referidos para evaluación de trasplante cardíaco por insuficiencia cardíaca refractaria a terapia médica. Fueron reclutados desde 1998 hasta 2004. Una vez estabilizados y bajo terapia médica óptima (drogas vasoactivas, diuréticos, vasodilatadores orales) quienes continuaban con hipertensión pulmonar (presión sistólica de arteria pulmonar (PSAP) $>40 \mathrm{mmHg}$ o resistencia vascular pulmonar $>3 \mathrm{UW}$ ) se incluyeron en el estudio. A todos los pacientes se les realizó monitorización hemodinámica invasiva (cateterismo de arteria pulmonar o sondeo cardíaco derecho) con medición de gasto cardíaco (GC), presión capilar pulmonar (PCP), resistencia vascular sistémica (RVS), resistencia vascular pulmonar (RVP) y presión arterial sistémica, presión arterial media (PAM), presión de arteria pulmonar sistólica y media (PAPm) y gradiente transpulmonar (GTP); todas éstas bajo el efecto de dosis repetidas de isosorbide sublingual de $5 \mathrm{mg}$ cada $5 \mathrm{~min}$ hasta conseguir disminución de RVP a $<3$ UW, PSAP $<40$ mmHg o hipotensión sintomática. Todos los resultados se expresan en promedio \pm desviación estándar. Los valores hemodinámicos basales y post dosis máximas de isosorbide se compararon utilizando test $t$ de Student.

\section{Resultados}

Se estudió a 21 pacientes, 18 hombres (85\%), edad promedio de $49 \pm 15$ años. La etiología de la insuficiencia cardíaca fue cardiopatía coronaria en 14 pacientes (67\%), miocardiopatía dilatada en 4 (19\%), enfermedad valvular en 3 (14\%). Cinco pacientes $(23 \%)$ se encontraban en capacidad funcional III y 16 (76\%) en capacidad funcional IV de la NYHA (Tabla 1). La fracción de acortamiento promedio del ventrículo izquierdo, medido por ecocardiografía, fue de $13 \pm 3 \%$. 
Tabla 1. Características de los pacientes

\begin{tabular}{|lr|}
\hline Número & 21 \\
Edad promedio & $49 \pm 15$ años \\
Hombres & $18(85 \%)$ \\
Etiología & \\
$\quad$ Cardiopatía coronaria & $14(67 \%)$ \\
$\quad$ Miocardiopatía dilatada & $4(19 \%)$ \\
Enfermedad valvular & $3(14 \%)$ \\
Insuficiencia cardíaca (clase NYHA) & \\
III & $5(33 \%)$ \\
IV & $16(77 \%)$ \\
\hline
\end{tabular}

La dosis máxima de isosorbide sublingual alcanzada fue de $15 \pm 5 \mathrm{mg}$. Luego de la administración de isosorbide se observó un descenso significativo en (Tabla 2): PAM (80 $\pm 8,5$ a $71 \pm 6,6$ mmHg, $p<0,0001)$ (Figura 1), PAPm $(38 \pm 11$ a $26 \pm 7,8 \mathrm{mmHg}, \mathrm{p}<0,0001)$, PSAP $(56 \pm 15$ a $38 \pm 11$ $\mathrm{mmHg})$, RVS (1.540 \pm 376 a $1.277 \pm 332$ dyn*s/cm5 p $<0,001)$, RVP $(3,5 \pm 2,2$ a $2,5 \pm 1,6$ UW, p $<0,05)$ (Figura 2) y GTP $(13 \pm 7$ a $10 \pm 4 \mathrm{mmHg}, \mathrm{p}<0,004)$. El gasto cardíaco aumentó de $3,96 \pm 0,7$ a 4,38 $\pm 0,9$ L/min, $\mathrm{p}=0,05$ (Figura 3) y la presión de capilar pulmonar disminuyó de $24 \pm 5$ a $16 \pm 4 \mathrm{mmHg}$. La

Tabla 2. Resultados pre y post isosorbide

\begin{tabular}{|lcc|}
\hline & Pre isosorbide & Post isosorbide \\
\hline PAM (mmHg) & $80 \pm 5$ & $71 \pm 6,6$ \\
PAPm (mmHg) & $38 \pm 11$ & $26 \pm 7,8$ \\
PSAP (mmHg) & $56 \pm 15$ & $38 \pm 11$ \\
RVS (dyn*s/cm5) & $1.540 \pm 376$ & $1.277 \pm 332$ \\
RVP (U Wood) & $3,5 \pm 2,2$ & $2,5 \pm 1,6$ \\
GTP (mmH) & $13 \pm 7$ & $10 \pm 4$ \\
GC (L/min) & $3,96 \pm 0,7$ & $4,38 \pm 0,9$ \\
PCP (mmHg) & $24 \pm 5$ & $16 \pm 4$ \\
\hline
\end{tabular}

$\mathrm{PAM}=$ presión arterial media; PAPm $=$ presión arteria pulmonar media; $\mathrm{PSAP}=$ presión sistólica arteria pulmonar; RVS= resistencia vascular sistémica; RVP= resistencia vascular pulmonar; GTP= gradiente transpulmonar; $\mathrm{GC}=$ gasto cardíaco; $\mathrm{PCP}=$ presión capilar pulmonar.

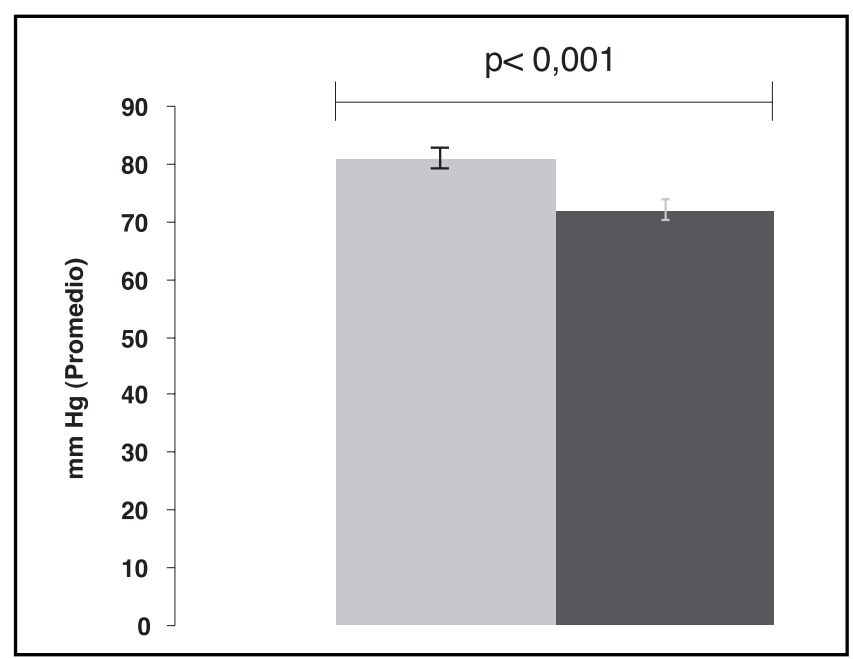

FiguRA 1. Presión arterial media pre y post isosorbide. Disminución en presión media post isosorbide. 


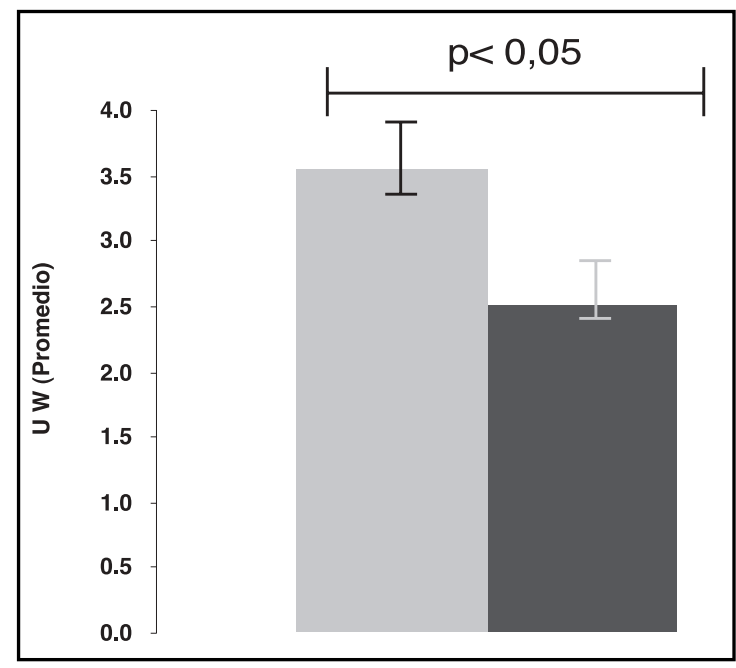

FIgURA 2. Resistencia vascular pulmonar pre y post isosorbide. Disminución en resistencia vascular pulmonar post isosorbide.

razón entre RVP/RVS antes y después de isosorbide fue 0,17 y 0,15 , respectivamente, $p=0,04$ (Figura 4).

En 19 pacientes (90\%) se logró una disminución de la RVP a valores $<4$ UW posterior a isosorbide. En dos pacientes se observó una disminución de la RVP sin llegar a cumplir los criterios de reversibilidad (de 5,9 a 4,1 UW y 7,35 a 4,33 UW, respectivamente).

Catorce pacientes fueron trasplantados (66\%) incluyendo el segundo paciente que presentaba reversibilidad parcial de la RVP. Sólo este paciente presentó insuficiencia cardíaca derecha post trasplante, la que ocurrió en las primeras $48 \mathrm{~h}$ de evolución y que respondió satisfactoriamente al tratamiento con milrinona intravenosa.

\section{DiscUSIÓN}

La administración de isosorbide sublingual es de utilidad en la evaluación de la reversibilidad de la hipertensión pulmonar en pacientes con insuficiencia cardíaca evaluados para trasplante cardíaco. El uso de isosorbide sublingual se asoció a una disminución significativa en la presión de arteria pulmonar y resistencia vascular pulmonar, en pacientes que se encontraban bajo una terapia

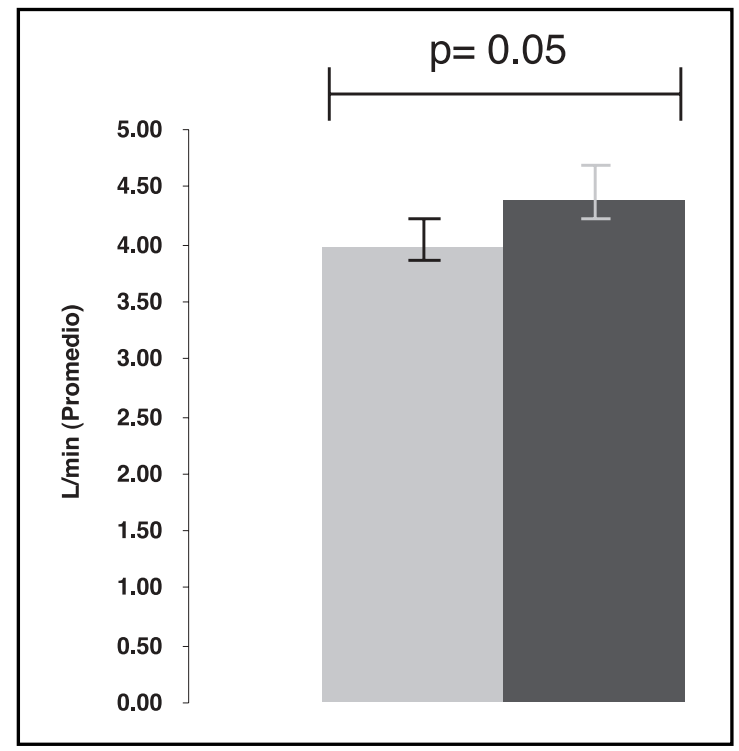

FigURA 3. Gasto cardíaco pre y post isosorbide. Mejoría en gasto cardíaco post isosorbide.

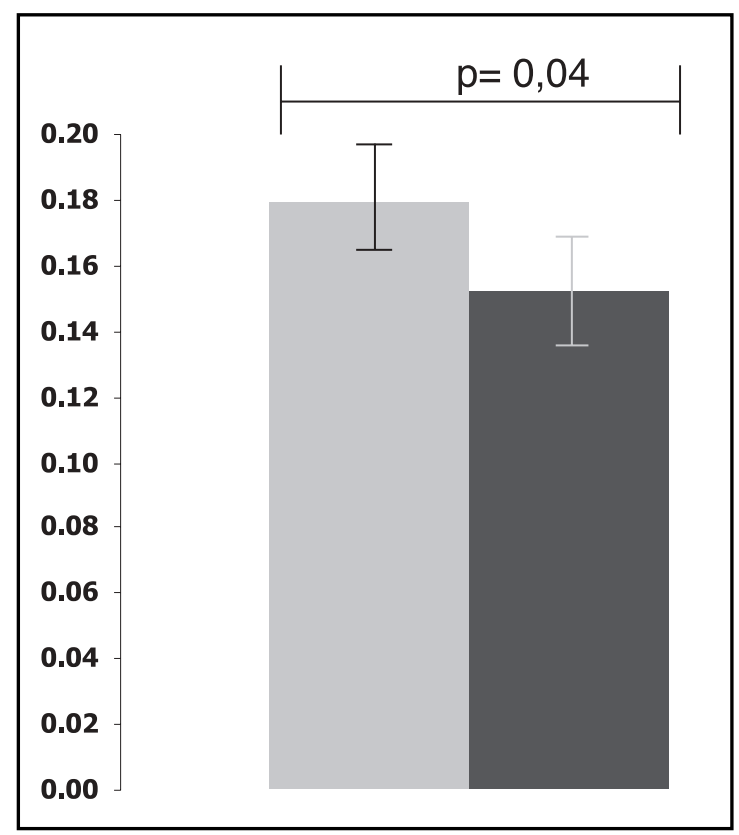

FIgURA 4. Razón RVP/RVS pre y post isosorbide. 
médica óptima, incluyendo en una alta proporción uso de inótropos y diuréticos intravenosos.

En la evaluación de la reversibilidad pulmonar se han ensayado diversas terapias, entre ellas, la más utilizada ha sido la administración intravenosa de nitroprusiato de sodio, un vasodilatador directo con efecto venoso y arterial. Se recomienda iniciar esta terapia con dosis de $0,5 \mu \mathrm{g} / \mathrm{kg} / \mathrm{min}$ e incrementarla gradualmente hasta conseguir un descenso en la resistencia vascular pulmonar o hipotensión sintomática. Si se logra reversibilidad de la RVP, es posible realizar el trasplante cardíaco con un menor riesgo de falla cardíaca derecha. En un estudio se evaluó la respuesta a nitroprusiato de sodio en pacientes con una RVP $>2,5$ UW $^{7}$. Estos pacientes fueron estratificados según la respuesta hemodinámica. Aquellos pacientes en que se logró disminuir la RVP a menos de 2,5 UW, tuvieron una mortalidad a los 3 meses post trasplante cardíaco de 3,8\%. Por el contrario, en aquellos pacientes en que la RVP no disminuyó bajo 2,5 UW o si sólo se logró a expensas de hipotensión (presión arterial sistólica $\leq 85 \mathrm{mmHg}$ ) la mortalidad fue mayor ( 40 y $27 \%$, respectivamente).

Otras alternativas terapéuticas utilizadas cuando la RVP o el GTP permanecen elevados es el empleo de otros vasodilatadores, como los derivados de las prostaglandinas (prostaciclina o PGE1) o la inhalación de óxido nítrico ${ }^{8,10}$. Sin embargo, estos tratamientos son de costo elevado y de administración compleja. Cuando el gasto cardíaco se encuentra disminuido en concomitancia con hipoperfusión o presión arterial baja el uso de inótropos como dobutamina en infusión, milrinona o levosimendan en bolo o infusión, en algunos casos asociados a dopamina u otros vasopresores, son de utilidad ${ }^{9,13}$.

La administración de isosorbide sublingual no ha sido descrita en la literatura. El isosorbide es un derivado de la nitroglicerina. Los nitratos son

\section{REFERENCIAS}

1. The Registry for the International Society for Heart and Lung Transplantation: Ninth official report. J Heart Lung Transplant 1992; 11: 599-606. poderosos venodilatadores que producen vasodilatación de los vasos coronarios epicárdicos, debido a este efecto directo, los nitratos pueden aumentar la función sistólica y diastólica por mejoría del flujo coronario en pacientes con cardiopatía isquémica $^{11,13}$. Aunque al igual que el nitroprusiato, disminuyen la presión arterial y la resistencia vascular sistémica, la reducción en las presiones de llenado es más prominente debido a su relativo mayor efecto en los vasos de capacitancia venosos. Estos efectos se asocian a una disminución del consumo miocárdico de oxígeno e incremento del débito cardíaco.

En nuestros pacientes se observó una reducción marcada en las presiones y resistencias vasculares pulmonares y sistémicas, asociados a una disminución de las presiones de capilar pulmonar y aumento del débito cardíaco. La disminución de la presión arterial fue bien tolerada y no se observó taquicardia, esto, probablemente, porque todos los pacientes se encontraban con presiones de llenados elevadas al inicio de la prueba.

La mejoría en la relación entre las RVP y sistémica permitiría observar un efecto vasodilatador predominante pulmonar del isosorbide y por consiguiente menores efectos sistémicos. Esto puede estar relacionado a una disminución de las presiones de capilar pulmonar y disminución de la vasoconstricción refleja secundaria.

En nuestra serie si bien es pequeña y no randomizada, la presencia de reversibilidad de la hipertensión pulmonar se asoció a una ausencia de falla ventricular derecha post trasplante.

A partir de la experiencia adquirida, en nuestro servicio esta prueba se utiliza como parte del protocolo de evaluación de trasplante cardíaco.

En resumen, la administración de isosorbide sublingual en dosis repetidas es una prueba útil, bien tolerada y sencilla de aplicar en la evaluación de la hipertensión pulmonar en pacientes potencialmente candidatos a trasplante cardíaco.

2. Tsai FC, Mareu $D$, Bresson J, Gjertson D, Kermani $R$, ARDEHALI A ET AL. Recent trends in early outcomes of adult patients after transplantation: a singleinstitution review of 251 transplants using standard donor organs. Am J Transplant 2002; 2: 539-45. 
3. Hosenpud JD, Bennet LE, Keck BM, Boucek MM, Novick RJ. The Registry for the International Society for Heart and Lung Transplantation: seventeenth official report. J Heart Lung Transplant 2000; 19: 909-31.

4. ERIKSON KW, Constanzo Nordin MR, O’SuLuvan EJ. Influence of preoperative transpulmonary gradient on late mortality after othotopic heart transplantation. J Heart Transplant 1990; 9: 52637.

5. Chen JM, Levin HR, Michler RE, Prusmack CJ, Rose EA, AARRONSON KD. Reevaluating the significance of pulmonary hypertension before cardiac transplantation: determination of optimal thresholds and quantification of the effect of reversibility on perioperative mortality. J Thorac Cardiovasc Surg 1997; 114: 627-34.

6. Wang SS, Chou NK, Hsu RB, Chen YS, Ko WJ, Yu HY ET AL. Heart-lung transplantation for severe pulmonary hypertension with severe heart failure: presentation of four cases. Transplant Proc 2003; 35: 450-2.

7. Costard-Jackie A, Fowler MB. Influence of preoperative pulmonary artery pressure on mortality after heart transplantation: testing of potencial reversibility of pulmonary hypertension with nitroprusside is useful in defining a high risk group. J Am Coll Cardiol 1992; 19: 48-54.
8. Mural S, Uretsky BF, Armitage JL, Tokarezyk TR, BETSCHART AR, Kormos RL ET AL. Utility of prostaglandin E1 in the pretransplantation evaluation oh heart failure patients with significant pulmonary hypertension. J Heart Lung Transplant 1992; 11: 716.

9. Givertz MM, Hare JM, Loh E, Gauthier DF, Colucci WS. Effect of bolus milrinone on hemodynamic variables an pulmonary vascular resistance in patients with severe left ventricular dysfunction: A rapid test for pulmonary hypertension. J Am Coll Cardiol 1996; 28: 1775.

10. Loh E, Stamler JS, Hare JM, Loscalzo J, Colucci WS. Cardiovascular effects of inhaled nitric oxide in patients with left ventricular dysfunction. Circulation 1994; 90: 2780.

11. Bundgaard H, Boesgaard S, Mortensen SA, ArenDRUP H, ALdERSHIVE J. Effect of nitroglycerin in patients with increased pulmonary vascular resistance undergoing cardiac transplant. Scand Cardiovasc J 1997; 31: 339-42.

12. Costard-Jacke A, Schroeder JS, Fowier MB. The influence of preoperative patient characteristics on early and late survival following cardiac trasplantation. Circulation 1991; 84 (suppl 3): 329-37.

13. ABRAMS J. Mechanisms of action of the organic nitrates in the treatment of myocardial ischemia. Am J Cardiol 1992; 70: 30B-42B. 